\title{
BRAIN
}

A JOURNAL OF NEUROLOGY

\section{The neural basis of impaired self-awareness after traumatic brain injury}

\author{
Timothy E. Ham, ${ }^{1,2}$ Valerie Bonnelle, ${ }^{3}$ Peter Hellyer, ${ }^{1}$ Sagar Jilka, ${ }^{1,4}$ Ian H. Robertson, ${ }^{5}$ \\ Robert Leech ${ }^{1}$ and David J. Sharp ${ }^{1}$
}

1 C3NL, Centre for Restorative Neuroscience, Division of Brain Sciences, Department of Medicine, Imperial College London, Hammersmith Hospital Campus, Du Cane Road, London, W12 0NN, UK

2 Systems and Restorative Neurology, University of Cambridge Neurology Unit, Herchel Smith Building for Brain and Mind Sciences, Robinson Way, Cambridge, CB2 OSZ, UK

3 Institute of Cognitive Neuroscience, University College London, Alexandra House, 17 Queen Square, London WC1N 3AR, UK

4 Department of Psychology, Goldsmiths, University of London, New Cross, London, SE14 6NW, UK

5 Trinity College Institute of Neuroscience, Trinity College Dublin, Dublin 2, Ireland

Correspondence to: Professor David Sharp,

The Computational,

Cognitive and Clinical Neuroimaging Laboratory,

Burlington Danes Building,

The Centre for Neuroscience,

The Division of Experimental Medicine,

Imperial College London,

Hammersmith Hospital Campus,

Du Cane Road, London,

W12 ONN, UK

E-mail: david.sharp@csc.mrc.ac.uk

Self-awareness is commonly impaired after traumatic brain injury. This is an important clinical issue as awareness affects longterm outcome and limits attempts at rehabilitation. It can be investigated by studying how patients respond to their errors and monitor their performance on tasks. As awareness is thought to be an emergent property of network activity, we tested the hypothesis that impaired self-awareness is associated with abnormal brain network function. We investigated a group of subjects with traumatic brain injury $(n=63)$ split into low and high performance-monitoring groups based on their ability to recognize and correct their own errors. Brain network function was assessed using resting-state and event-related functional magnetic resonance imaging. This allowed us to investigate baseline network function, as well as the evoked response of networks to specific events including errors. The low performance-monitoring group underestimated their disability and showed broad attentional deficits. Neural activity within what has been termed the fronto-parietal control network was abnormal in patients with impaired self-awareness. The dorsal anterior cingulate cortex is a key part of this network that is involved in performance-monitoring. This region showed reduced functional connectivity to the rest of the fronto-parietal control network at 'rest'. In addition, the anterior insulae, which are normally tightly linked to the dorsal anterior cingulate cortex, showed increased activity following errors in the impaired group. Interestingly, the traumatic brain injury patient group with normal performance-monitoring showed abnormally high activation of the right middle frontal gyrus, putamen and caudate in response to errors. The impairment of self-awareness was not explained either by the location of focal brain injury, or the amount of traumatic axonal injury as demonstrated by diffusion tensor imaging. The results suggest that impairments of self-awareness after traumatic brain injury result from breakdown of functional interactions between nodes within the fronto-parietal control network. 
Keywords: traumatic brain injury; fronto-parietal control network; salience network; self-awareness; functional connectivity Abbreviations: $F P C N=$ fronto-parietal control network; $P M=$ performance monitoring; $T B I=$ traumatic brain injury

\section{Introduction}

Traumatic brain injury (TBI) frequently produces impairments of awareness (Prigatano and Altman, 1990; Vanderploeg et al., 2007). These can range from coma to more subtle problems with self-awareness, which are common in the chronic stages of the disease (Prigatano and Altman, 1990; Fleming et al., 1996; O'Keeffe et al., 2007). Persistently impaired self-awareness can be a major clinical problem as it potentially limits rehabilitation (Sherer et al., 2005), and is associated with poor functional outcomes (Sherer et al., 2003; O'Keeffe et al., 2007). An objective measure of self-awareness can be obtained by studying patients' abilities to monitor and respond to their own errors (Hart et al., 1998; O'Keeffe et al., 2004; Modirrousta and Fellows, 2008; Ornstein et al., 2009). Healthy adults normally rapidly identify and correct their own errors (Rabbitt, 1966, 1968), whereas impairments in performance monitoring are common after TBI (Larson et al., 2007; Ornstein et al., 2009).

Recent work has clarified the brain regions involved in performance monitoring (Ullsperger and von Cramon, 2004; Ham et al., 2012). Structures within the medial prefrontal cortex, particularly the dorsal anterior cingulate cortex, respond to many types of errors (Dehaene et al., 1994; Ridderinkhof et al., 2004; Sharp et al., 2006) and seem to be necessary for rapid on-line error processing (Modirrousta and Fellows, 2008). The dorsal anterior cingulate cortex is tightly linked, both structurally and functionally, to the anterior insulae, which together are often referred to as the salience network (Seeley et al., 2007; Ullsperger et al., 2010; Ham et al., 2013). These regions also form part of a larger frontoparietal control network (FPCN), which includes bilateral inferior frontal gyri and inferior parietal lobes (Vincent et al., 2008; Spreng, 2012). Activity within the salience network is linked post-error slowing ( $\mathrm{Li}$ et al., 2008), and we have previously shown that changes in the interactions between the dorsal anterior cingulate cortex and insulae correlate with the magnitude of this behavioural adaptation (Ham et al., 2013). One prominent theory proposes that the insulae encode neural representations of aspects of the 'self'. This is updated moment-to-moment by input from the dorsal anterior cingulate cortex, allowing the integration of motivation, social and cognitive processing (Craig, 2009).

Right frontal and parietal lobe damage after stroke commonly produces spatial neglect (Husain et al., 1997; Corbetta and Shulman, 2011). This is associated with reduced interaction between frontal and parietal regions (functional connectivity), consistent with awareness being an emergent property of longdistance interactions between brain regions (Mesulam, 1990; Taylor, 1997; He et al., 2007). In contrast, relatively little is known about the neural basis of impaired self-awareness after TBI. The location and extent of focal brain injuries are not reliable predictors of self-awareness impairment (Sherer et al., 2005). This may reflect the importance of network disruption as a causative mechanism, which until recently has been difficult to study
(Sharp and Ham, 2011; Ham and Sharp, 2012). The one previous functional MRI study provides preliminary evidence that selfawareness impairment after TBI may be associated with abnormal activity in the right prefrontal cortex (Schmitz et al., 2006).

We investigated for the first time whether self-awareness deficits are associated with network dysfunction after TBI. Two groups of patients with high and low levels of performance monitoring (high-PM/low-PM) were carefully defined on the basis of their ability to correct errors made on a simple cognitive task. This measure has previously provided a robust 'on-line' measure of performance monitoring (O'Keeffe et al., 2007; Modirrousta and Fellows, 2008). Given the prominence of dorsal anterior cingulate cortex and insulae responses to errors, their key role within an extended FPCN, and the importance of functional disconnection in the aetiology of spatial neglect, we predicted that a breakdown of functional connectivity within the FPCN would be observed in patients with impaired performance-monitoring. Therefore, we focused our analysis on the FPCN, and tested the specific hypothesis that impaired performance monitoring is associated with altered functional connectivity within the FPCN. Advanced multimodal neuroimaging allowed investigation of the structure and function of this network in the absence of a specific task ('rest'), and during error processing. Functional MRI was used to measure the interactions between nodes in these networks and their response to errors. Diffusion tensor imaging and structural MRI were used to assess the integrity of brain regions and structural connections within the networks.

\section{Materials and methods}

\section{Patients demographic and clinical details}

Seventy-three patients with TBI were recruited from a neurology clinic where they had been referred for persistent neurological symptoms. Patients with a range of injury severities were recruited to provide variability of cognitive disabilities. Ten patients were excluded: two had distorted magnetic resonance images; six could not perform the cognitive tasks adequately; and two had premorbid psychiatric illness. The 63 patients included in the main analysis ( 46 males, mean age $38.0 \pm 12$ years, range 18-66 years) were in the post-acute/chronic phase post-injury (mean $29 \pm 74$ months, range 2-578 months) (see Supplementary Table 1 for clinical details).

TBI severity was assessed according to the Mayo Classification (Malec et al., 2007). This integrates the duration of loss of consciousness; length of post-traumatic amnesia; lowest recorded Glasgow coma scale in the first $24 \mathrm{~h}$; and neuroimaging. Using this system 89\% (56 patients) were classified moderate/severe; $8 \%$ (five patients) mild (probable); and 3\% (two patients) symptomatic (possible) TBI. All patients with impaired performance monitoring were of moderate/ severe severity. Exclusion criteria included neurosurgery, except for invasive intracranial pressure monitoring $(n=1)$; psychiatric or 


\section{A Patient classification}

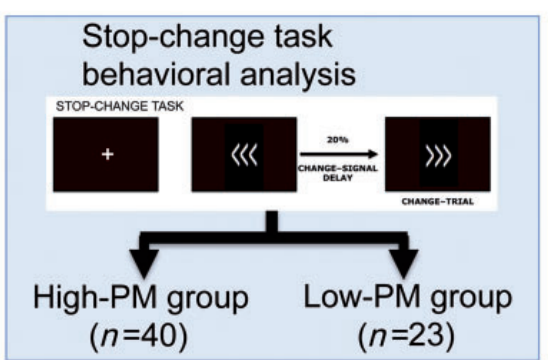

B Structural assessment

DTI and Lesion masks

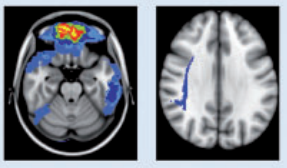

Comparison of white and grey matter damage

\section{Reference network definition}

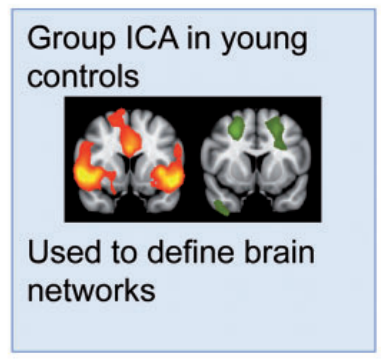

D Resting-state functional connectivity

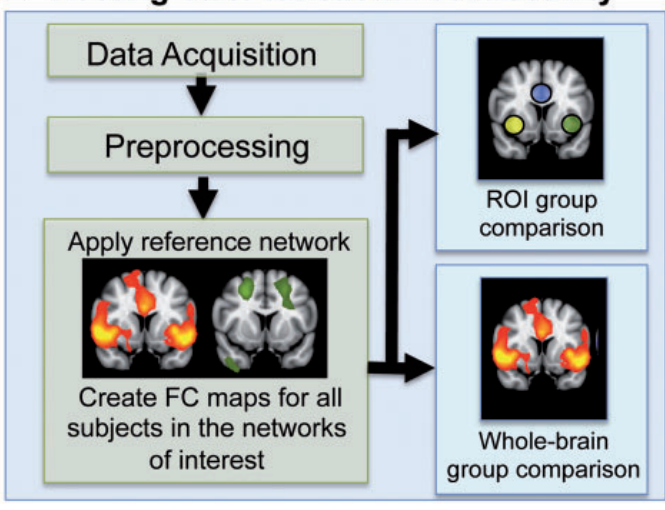

E Standard GLM fMRI analysis

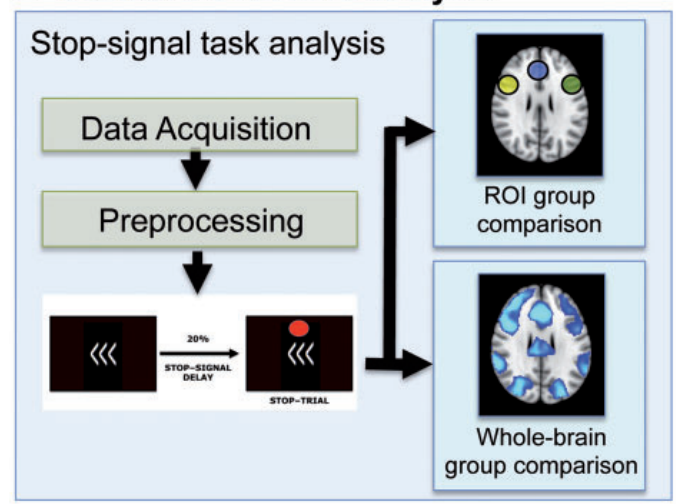

Figure 1 A high-level description of the processing steps for behavioural, structural, rest and task functional MRI data analysis. (A) Behaviour on the stop-change task was used to identify patients with high and low levels of PM. (B) Traumatic brain injury was assessed structurally by mapping the location of focal injuries using high-resolution $T_{1} M R I$, and the location of traumatic axonal injury using diffusion tensor imaging. (C) Reference fronto-parietal control and visual networks were defined from young control subjects. (D) These networks were used to investigate functional connectivity in 'resting' state functional MRI data (fMRI). (E) Brain activation in response to errors was assessed using functional $M R I$ data acquired during performance of the stop-signal task. DTI = diffusion tensor imaging; ICA = independent component analysis; $F C=$ functional connectivity; $G L M=$ general linear model; $R O I=$ region of interest.

neurological illness before their head injury; significant previous TBI; current or previous drug or alcohol abuse; or contraindication to MRI. Subjects had no neurological, major medical or psychiatric disorders before TBI. All participants gave written consent. Hammersmith and Queen Charlotte's and Chelsea research ethics committee approved the study.

\section{Standard clinical imaging}

Patients were assessed using standard $T_{1}$ and $T_{2}$ FLAIR MRI, and gradient echo imaging to improve sensitivity to micro-bleeds, a surrogate marker of diffuse axonal injury (Scheid et al., 2003). A senior neuroradiologist reviewed MRI scans. Abnormalities on initial CT imaging or follow-up MRI were seen in $89 \%$ of patients. Of the 51 initial CT reports available, $56 \%$ had at least one contusion; $53 \%$ had skull fractures; $33 \%$ had subarachnoid haemorrhages; $25 \%$ had subdural haematomas; $24 \%$ had extra-dural haematomas; and $6 \%$ had evidence of cerebral oedema. As expected, many patients had multiple abnormalities on standard clinical MRI performed for the study (Supplementary Table 1).

\section{Neuropsychological assessment}

A battery of neuropsychological tests assessed the type of cognitive dysfunction commonly experienced after TBI. This included assessment of premorbid intelligence, current verbal and spatial reasoning, processing speed and verbal fluency (Supplementary material). A subset of patients $(n=39)$ completed the Hospital Anxiety and Depression Scale assessmens. Both patients and their relatives completed the Frontal Systems Behaviour questionnaire (Grace et al., 1999). The Frontal Systems Behaviour questionnaire provides a measure of pre$\mathrm{TBI}$ and post-TBI dysexecutive symptoms assessed by the patient and their relative. The disparity between observers' and patients' scores provides a 'meta-cognitive' measure of self-awareness (Reid-Arndt et al., 2007), which we compared to the 'on-line' measure derived from the stop-change task (see below).

\section{Experimental overview}

A high-level description of our methods is provided in Fig. 1.

\section{Control groups}

Separate control groups were used for separate branches of the study (Fig. 1). One group of control subjects was used to define the FPCN and a visual network in an unbiased way (see below) (19 subjects, 12 male, mean age $24.5 \pm 2.75$ years). A further four groups were used to assess: (i) neuropsychological function (20 subjects, 11 male, mean age $35.75 \pm 11$ years); (ii) stop-change task behaviour (11 subjects, four males, mean age $30.4 \pm 7.7$ years) (Fig. $1 \mathrm{~A}$ ); (iii) resting state functional connectivity and diffusion tensor imaging analyses 
(24 subjects, 16 males, mean age $36.2 \pm 10.2$ years) (Fig. $1 B$ and D); and (iv) stop-signal task functional MRI (25 subjects, 17 males, mean age $34.8 \pm 9.6$ years) (Fig. $1 \mathrm{E}$ ).

\section{Behavioural assessment: stop-change task}

Performance monitoring was investigated behaviourally using the stop-change task, which was performed outside the scanner to define with high-PM and low-PM groups (Fig. 1A). The stop-change task is a timed choice-reaction task where subjects respond to an arrow cue with either a right or left button press (Bekker et al., 2005; Verbruggen and Logan, 2008). On an unpredictable subset of trials subjects are signalled to withhold their initial response and respond with the alternate button press by a switch in the direction of the arrow (change-trials) (Fig. 2A). The delay between the initial presentation of the arrow and the change of its direction varied using a staircase procedure to produce an error rate of $\sim 50 \%$ in each subject. If subjects fail to inhibit their initial response they are instructed to correct themselves by subsequently pressing the correct button (Supplementary material). This correction response provides a rapid on-line performance-monitoring measure, as it will only be made if subjects are aware of making an error. Patients were split into lowPM and high-PM groups based on the number of errors they corrected on the stop-change task compared to healthy control subjects (see 'Results' section).

\section{Structural and functional magnetic resonance imaging acquisition}

Anatomical and functional MRI was acquired using standard procedures (Supplementary material). The majority of patients were assessed in two sessions: session one involved task-based functional MRI, acquired whilst they performed the stop-signal task; session two involved resting-state functional $M R I, T_{1}$, gradient echo and diffusion tensor imaging. During the resting-state functional MRI patients were instructed to close their eyes and try to relax.

\section{Functional connectivity analysis of 'resting' brain networks}

We used a dual regression, independent component analysis to study functional connectivity in resting-state functional MRI (Zuo et al., 2010) (Fig. 1C and D). This provides a voxel-wise measure of functional connectivity that reflects the correlation between the activity of each voxel and the rest of the network being tested. The first step involved generating reference brain networks based on 19 healthy control subjects who were not included in subsequent analyses. A temporal concatenation independent component analysis was performed on the healthy control subjects' resting-state data, with the number of components constrained to facilitate comparison with previous work (Damoiseaux et al., 2006). The probabilistic independent component analysis output included 11 well-recognized brain networks, as well as components that represented physiological noise and movement artefacts (Beckmann et al., 2005; Smith et al., 2009) (see Supplementary Fig. 1 for individual networks). Because of its involvement in performance monitoring we focused our analysis on the FPCN component, which contained the dorsal anterior cingulate cortex and anterior insulae as well as other frontal and parietal regions (Vincent et al., 2008; Spreng et al., 2010) (Supplementary Fig. 1A and Supplementary Table 3). In addition, we investigated a visual network, where we did not expect to see major functional abnormalities, to demonstrate that any effects were specific to the FPCN. The visual

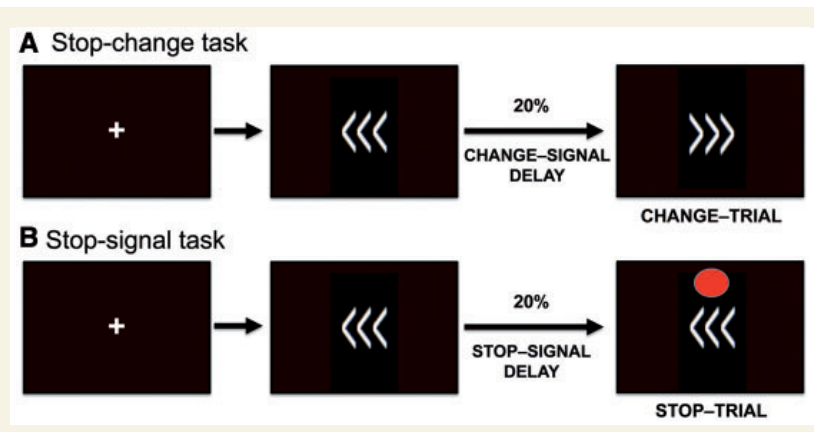

Figure 2 Schematic representation of the (A) stop-change task and the (B) stop-signal task. Both tasks involved the presentation of a fixation cross, followed by arrows pointing to the right or left. Subjects respond to the direction of the arrow with an appropriate finger press. Twenty per cent of the stop-change task trials were 'change-trials' where the arrows changed direction after a short delay. Subjects had to respond to the final direction of the arrows. Twenty per cent of the stop-signal task trials were 'stop-trials' where a red dot appeared after a short delay indicating that subjects were to inhibit all responses. The timing of the stop and change cues both varied adaptively to generate an error rate of $\sim 50 \%$.

network included occipital regions and regions within the dorsal and ventral visual pathways in the superior parietal and inferior temporal lobes (Supplementary Fig. 1B and Supplementary Table 3).

Functional connectivity was compared in the two patient groups and a control group as follows: (i) the networks chosen from the independent component analysis were used as weighted seed regions to derive individual time courses for those regions for each subject; and (ii) these time courses were then re-regressed onto each subject's data resulting in a subject-specific spatial map of functional connectivity (Filippini et al., 2009). Spatial maps were tested for voxel-wise betweengroup differences using non-parametric permutation testing (Smith et al., 2004). Voxel-wise estimates of the probability of grey matter membership intensity were included as a covariate to control for the effects of atrophy and cortical damage associated with TBI. To correct for multiple comparisons, results were cluster corrected using threshold-free cluster extraction and a family-wise error rate of $<0.05$.

In addition to the voxel-wise analyses, regions of interest were used to probe the relationship between performance-monitoring and functional connectivity in theoretically motivated nodes of the FPCN (Fig. 1D). To avoid bias, we used peaks of the independent component analysis derived networks defined from the young control subjects. Peaks from the FPCN and visual network were used to define the centre of $10 \mathrm{~mm}$ diameter spherical region of interests (Supplementary Table 3). The FPCN was used to define the dorsal anterior cingulate cortex, right insula, and left insula. The visual network was used to define regions in bilateral occipital lobe and precuneus.

\section{Stop-signal task}

We investigated error processing in patients with high and low performance-monitoring abilities in a subgroup of 48 patients using functional MRI analysis of the stop-signal task (37 males, mean age $35.7 \pm 10.9$, range $18-54$ years) (Fig. 1E). The stop-signal task allowed us to investigate phasic responses to behaviourally salient events. For methodological reasons we did not use the stop-change 
task in the scanner. When subjects are aware of an error on the stopchange task and correct it, they make two motor responses. In contrast, when subjects are unaware of an error they make only one response. The effect of different numbers of motor responses on the FPCN and other networks cannot be easily separated in functional MRI studies. Therefore, in the scanner we used a simpler variant of the stop-change task, the stop-signal task (Logan et al., 1984) (Fig. 2B). The stop-signal task is designed to produce a fixed number of errors in all subjects, and we have previously used it to investigate attention, response inhibition and error processing in healthy control subjects and patients with TBI (Sharp et al., 2010; Bonnelle et al., 2011, 2012). Like the stop-change task, an arrow signals the direction of response on go-trials. On an unpredictable subset of trials, a stop-signal appears and subjects have to withhold but not change their response (stoptrials) (Supplementary material). Like the stop-change task the delay between the appearance of the arrow and the stop-signal varied to produce errors on $\sim 50 \%$ of trials. Unlike the stop-change task, the stop-signal task only requires response inhibition, rather than response inhibition and correction.

\section{Functional magnetic resonance imaging analysis of the stop-signal task}

Standard functional MRI analysis was performed using FMRIB Software Library (Supplementary material). Lesion maps were used to improve brain registration to standard space (Brett et al., 2001). The following contrast images were generated: incorrect stop-trial versus go-trials and correct stop-trials versus go-trials. The two stopsignal task runs were first analysed separately and then combined by using fixed effects analysis. Group effects were analysed by using FLAME [Oxford Centre for Functional MRI of the Brain (FMRIB) Local Analysis of Mixed Effects]. Mean changes in activation patterns were then compared between groups. The final statistical images were thresholded by using Gaussian random field-based cluster inference with a height threshold of $Z>2.3$ and a cluster significance threshold of $P<0.05$. Individual grey matter density maps were included in the FMRI Expert Analysis Tool (FEAT) GLM as a confound regressor (Supplementary material) (Oakes et al., 2007).

\section{Structural lesion analysis}

We next investigated whether the pattern of focal injury or traumatic axonal injury related to impaired self-awareness (Fig. 1B).

\section{Focal lesions}

Cortical lesions were defined manually on high-resolution $T_{1}$ images using FSLview. White matter and cortical lesions were registered to a standard MNI $152 \mathrm{~T}_{1} 1 \mathrm{~mm}$ template using FLIRT (Jenkinson and Smith, 2001; Jenkinson et al., 2002). Lesion overlap images were then created and compared using FSLmaths.

\section{Structural connectivity: diffusion tensor imaging}

Diffusion tensor imaging analysis was performed to test whether self-awareness problems after TBI were associated with white matter damage within the FPCN. Sixty-four direction diffusion tensor images were acquired (Supplementary material) and standard diffusion tensor imaging preprocessing methods were used with the FMRIB software library (Smith et al., 2004). We then used probabilistic tractography to create masks of the white matter tracts connecting peak regions within the reference networks. Tracts within the FPCN connected dorsal anterior cingulate cortex to right and left insula and tracts within the visual network connected the right and left precuneus to the respective lateral occipital cortices (Supplementary material). The white matter skeleton produced by Tract Based Spatial Statistics (Smith et al., 2006) constrained the analysis of white matter to the central parts of white matter tracts. Although this results in an incomplete assessment of the white matter, it provides major methodological advantages such as minimizing partial-volume errors. We have shown that constraining the sampling of white matter damage by the white matter skeleton more accurately estimates white matter integrity after TBI than alternative approaches (Squarcina et al., 2012). Using these masks, translated into each subject's brain space, fractional anisotropy values for each tract of interest were determined.

\section{Results}

\section{Using the stop-change task to define self-awareness}

During the stop-change task subjects were instructed to correct any perceived errors, providing an explicit measure of 'on-line' performance monitoring. Our control group consistently corrected their errors during the stop-change task $(98.4 \pm 0.96 \%)$, demonstrating that errors on this task are usually easily identified and corrected. In contrast, patients with TBI were highly variable in the proportion of errors they corrected. Most patients $(n=40)$ had a similar range to control subjects for error correction $[<2$ standard deviations (SD) from the mean of the control group]. These patients formed the high-PM group. In contrast, a significant minority of patients $(n=23)$ performed outside this range, correcting significantly fewer errors than control subjects $(68.8 \pm 5.6 \%)$. These patients formed our low-PM group (Fig. 3A).

The Low-PM group showed abnormal performance on other aspects of the stop-change task in addition to the number of errors they corrected. The time taken for the low-PM group to correct an error was significantly slower than the high-PM group (420 versus $278 \mathrm{~ms}, \mathrm{t}=5.73, d f=61, P<0.001$ ) and control subjects (420 versus $250 \mathrm{~ms} ; \mathrm{t}=4.30, d f=32, P<0.001$ ); as was the reaction time on go-trials compared to high-PMs (533 versus $387 \mathrm{~ms} ; \mathrm{t}=6.34, d f=61, P<0.001)$ and control subjects $(533$ versus $400 \mathrm{~ms} ; \mathrm{t}=3.47, d f=31, P=0.002$ ). Intra-individual variability of reaction times on go-trials was also significantly greater in the low- than the high-PM group $(0.227$ versus $0.174 ; \mathrm{t}=4.33$, $d f=61, P<0.001)$ and control subjects $(0.227$ versus 0.155 ; $\mathrm{t}=3.78, d f=32, P=0.001$ ). In contrast, the high-PM group did not differ significantly from control subjects in any of these measures. These results are consistent with impaired performance monitoring being part of a more general attentional deficit in the low-PM group.

In other ways, the high- and low-PM groups were similar. The groups performed equally well on tests of memory, verbal and nonverbal reasoning. Both groups had similar scores on the Hospital Anxiety and Depression Scale questionnaire assessment of anxiety and depression. There was also no significant difference between groups in: (i) age, [low-PM $39.4 \pm 12.8$ years (range 20-67 years) versus high-PM $37.3 \pm 11.6$ years (range 18-56 years)]; (ii) time since injury [low-PM $46.9 \pm 115.3$ months (range 2-563 months) 
A

Error correction during the stop-change task

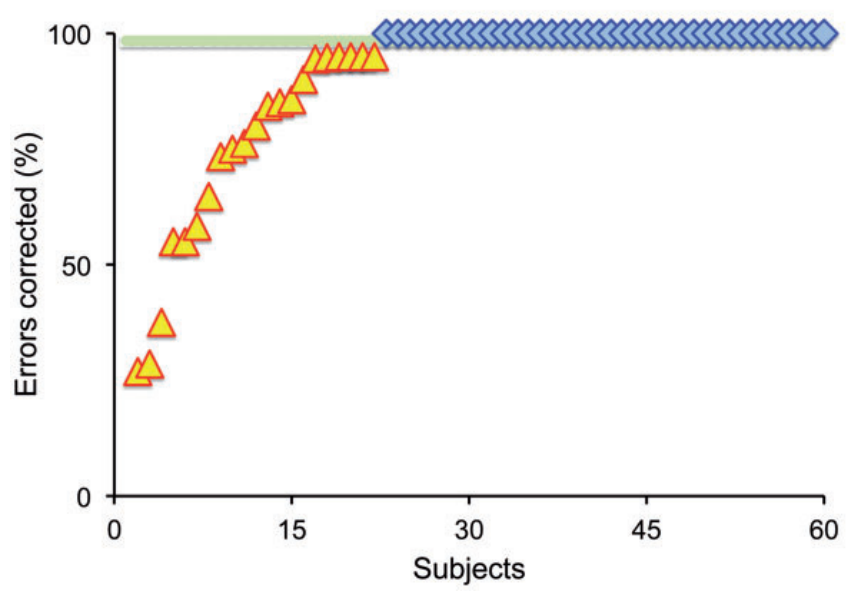

B Frontal systems behavior scale

(FrSBe) scores

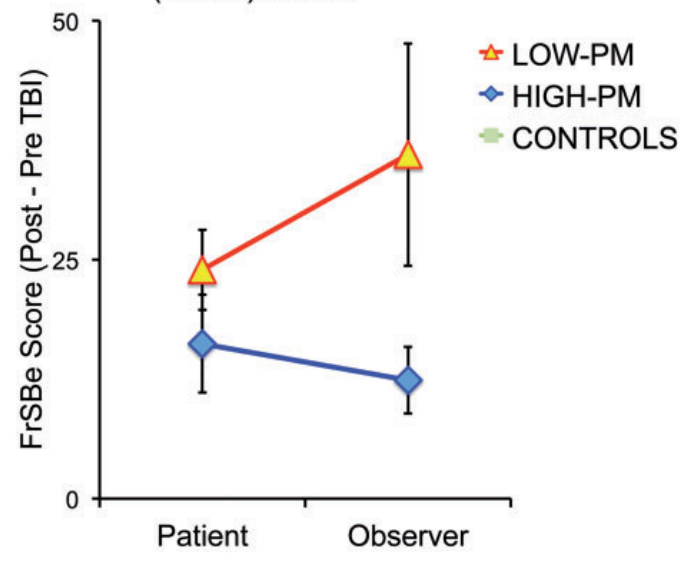

Figure 3 Impairments of self-awareness demonstrated by abnormal performance monitoring and meta-cognitive self-assessment. (A) The percentage of stop-change task errors corrected by individuals in the control (green), high-PM (blue) and low-PM (yellow) groups. (B) Changes in Frontal Systems Behavioural (FrSBe) questionnaire score after TBI in the high- and low-PM groups, as assessed by the subjects themselves and an observer who knew the patient well. Error bars indicate SEM.

versus high-PM $19.7 \pm 30.1$ months (range 2-168 months)]; or (iii) injury severity (low-PM 100\% moderate/severe versus high-PM $82.5 \%$ moderate/severe). There were significantly more males in the high-PM group than the low-PM group (59\% versus $82 \%$, $\chi^{2}=5.0, d f 1, P=0.025$ ) (Supplementary Table 1). The subgroup of patients $(n=48)$ who had functional MRI consisted of low-PM $(n=18)$ and high-PM patients $(n=30)$. These groups were also matched for age, gender, time since injury, and injury severity.

\section{Post-error slowing on the stop-change task}

Importantly, the low-PM group did not show post-error slowing after errors they failed to correct. This supports the inference that low-PM patients were unaware of their uncorrected errors. Conversely, compared to the high-PM group they showed greater post-error slowing after correct errors ( 86 versus $48 \mathrm{~ms} ; \mathrm{t}=2.94$, $d f=59, P=0.005)$, and a trend towards greater post-error slowing compared with control subjects (86 versus $49 \mathrm{~ms} ; \mathrm{t}=1.79$, $d f=29.7, P=0.084$ ) (Supplementary material). The increase in post-error slowing after corrected errors indicates that the lowPM group were engaged in the task, and were able to appropriately adapt to errors if they were initially recognized. More generally, the low-PM group also performed more poorly than the high-PM group and control subjects on several tasks of processing speed and executive function (Supplementary Table 2).

\section{Impaired error processing is a marker of more general self-awareness problems}

The Frontal Systems Behaviour questionnaire provided additional evidence that the low-PM group had a general impairment of selfawareness (Fig. 3B). Both patient groups reported similar levels of disability on the Frontal Systems Behaviour questionnaire.
However, the carers of the low-PM group made more severe assessments of severity than the carers of high-PM patients $(P=0.01)$, providing evidence for a general impairment of selfawareness in the low-PM group.

\section{Impaired performance monitoring is associated with reduced functional connectivity within the fronto-parietal control network}

The low-PM group showed reduced functional connectivity within the FPCN, compared with the high-PM group and control subjects. Whole-brain analysis comparing patient groups showed the dorsal anterior cingulate cortex, right inferior frontal gyrus, and right middle frontal gyrus were significantly less functionally connected to the rest of the FPCN in the low-PM group (Fig. 4A and Supplementary Table 4). A targeted region of interest analysis also assessed the main nodes of the FPCN and visual network. This revealed that the low-PM group also showed reduced dorsal anterior cingulate cortex functional connectivity compared with control subjects $(\mathrm{t}=-2.3, d f=45, P<0.028$ ), although this result was not significant on whole-brain analysis (Fig. 4B). In contrast, no other nodes within the FPCN showed reduced functional connectivity in low-PM patients, and there were no abnormalities in the functional connectivity of the visual network on whole-brain or region of interest analysis (Fig. 4C).

\section{Stop-signal task functional magnetic resonance imaging analysis}

\section{Stop-signal task behavioural results}

Overall, the groups performed the tasks well. The low- and highPM groups were equally accurate on stop (49.8\% versus $52.0 \%$ ) 


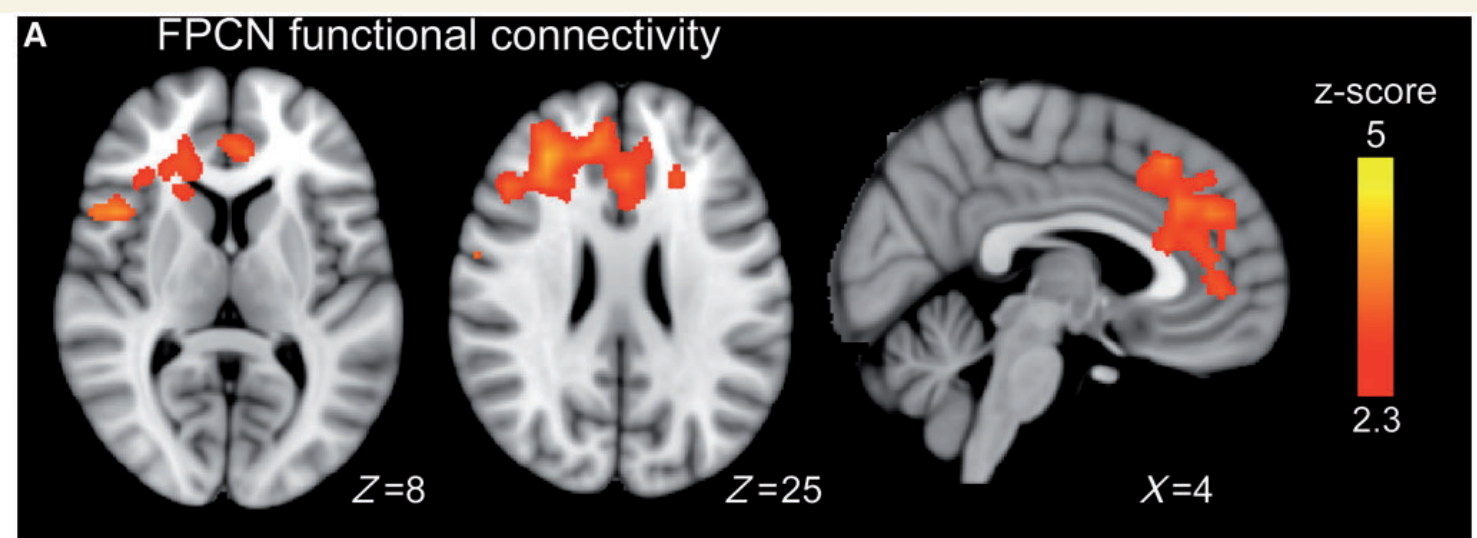

B

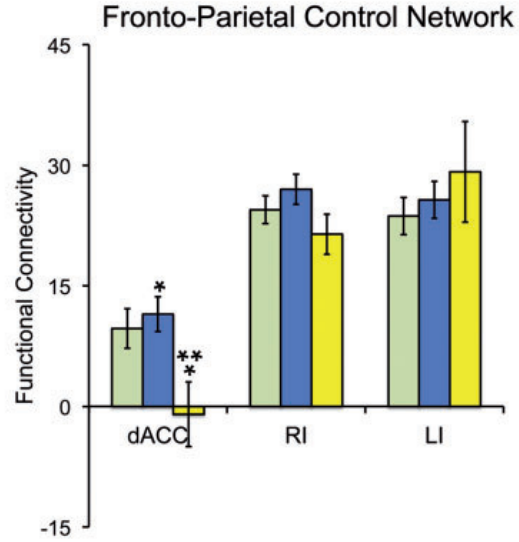

c

C Visual Network

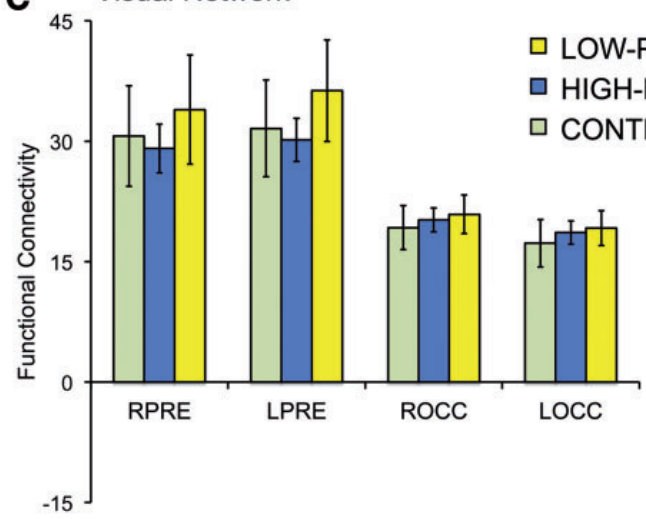

Figure 4 Impaired performance monitoring is associated with reduced functional connectivity of the dorsal anterior cingulate cortex. (A) Comparison of the functional connectivity within the FPCN of the high-PM and low-PM groups. The image was thresholded using threshold free cluster enhancement (TFCE) (family-wise error correction $<0.05$ ). (B and C) Region of interest analyses comparing the functional connectivity of key regions within the FPCN (B), and control visual network (C) across the three subject groups. ${ }^{*} P<0.05$ significant difference between the high and low-PM groups. ${ }^{*} P<0.05$ significant difference between the low-PM and healthy control groups. Error bars indicate SEM. Regions of interest were the dorsal anterior cingulate cortex (dACC), right insula (RI), left insula (LI), left precuneus (LPRE), right precuneus (RPRE), left occipital lobe (LOCC) and right occipital lobe (ROCC).

and go-trials (97.8\% versus $96.7 \%$ ), and these accuracies were not significantly different to control subjects. Similar to their performances on the stop-change task, the low-PM group was slower (624 versus $460 \mathrm{~ms} ; \mathrm{t}=4.37, d f=46, P<0.001$ ), with greater intra-individual variability on go-trials compared with the highPM group ( 0.210 versus $0.174 ; \mathrm{t}=2.58, d f=46, P=0.013)$ and control subjects (0.210 versus $0.170 ; \mathrm{t}=3.51, d f=40, P=0.001$ ). Interestingly, the low-PM group showed greater post-error slowing following errors on the stop-signal task than the high-PM group (87 versus $32 \mathrm{~ms} ; \mathrm{t}=3.01, d f=46, P=0.004$ ) and control subjects ( 87 versus $25 \mathrm{~ms} ; \mathrm{t}=3.06, d f=40, P=0.004$ ). This was similar to the enhanced post-error slowing observed after corrected errors in the stop-change task, and suggests that the low-PM group was aware of at least a proportion of these errors.

\section{Impaired performance monitoring is associated with increased insulae activation after errors}

The direct contrast between neural activity on incorrect stop-trials and go-trials allows an investigation of the neural correlates of error processing. As expected, errors were associated with activation in a network of regions including the dorsal anterior cingulate cortex, bilateral insulae, the frontal poles, lateral prefrontal cortex, and supramarginal gyrus in all groups (Supplementary Fig. 2). Whole-brain analysis revealed that the low-PM group showed greater activation than control subjects in both bilateral insulae and parietal operculum in response to errors (Fig. 5A and Supplementary Table 4). A similar effect was seen when comparing low- and high-PM groups, although the increases in insula and parietal operculum activation were lateralized to the left hemisphere (Fig. 5B and Supplementary Table 4). These abnormal activity patterns overlapped with the FPCN defined from analysis of 'resting' functional MRI (blue background Fig. 5). Dorsal anterior cingulate cortex activation after errors was similar across the groups. No regions showed increased activity in the highPM group or control subjects compared with the low-PM group after errors.

Normal performance monitoring after traumatic brain injury associated with increased activation in the right middle frontal gyrus

The contrast of incorrect stop-trials and go-trials showed the highPM group had greater activation than control subjects in the right 


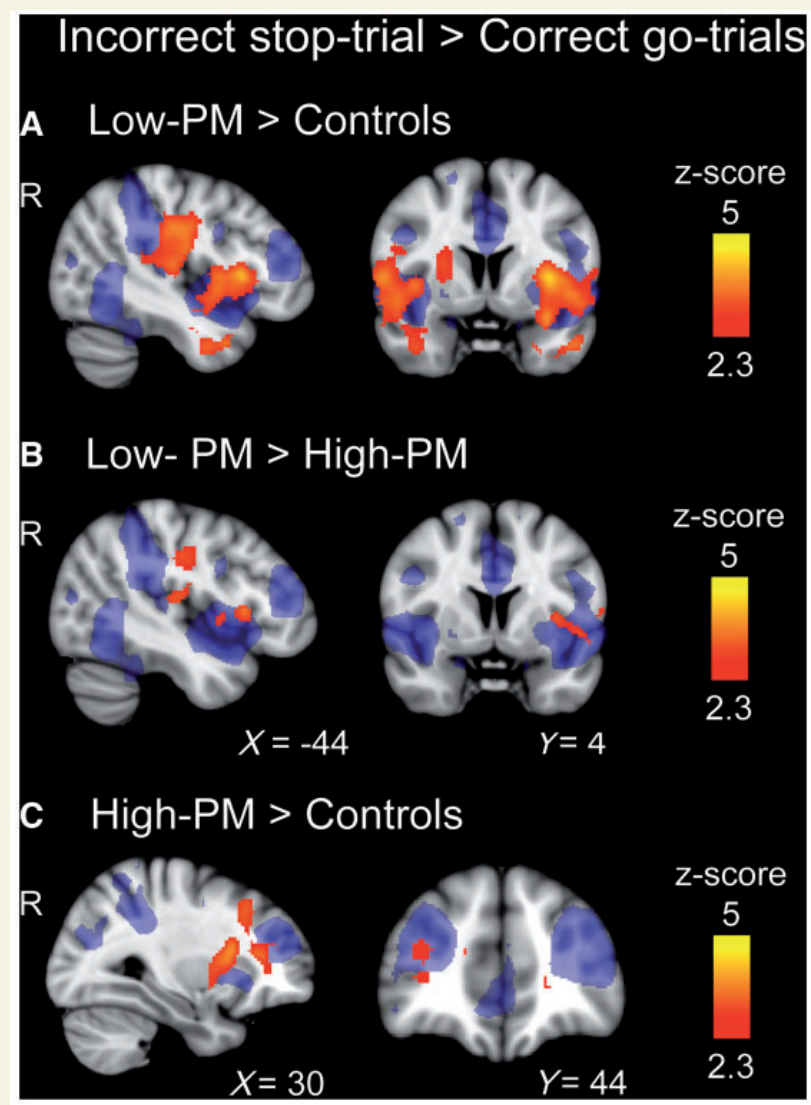

Figure 5 Abnormal error-related activity in the FPCN in TBI patients. Group contrasts of brain activation after errors on the stop-signal trial (incorrect stop-trials versus correct go-trials). The figures are comparisons between: (A) low-PM patients > controls; (B) low-PM > high-PM patients; and (C) high-PM patients $>$ controls. Areas with greater activation are shown red-yellow. All contrasts are superimposed on an image of the FPCN (blue) for reference. Images are cluster corrected (z-statistic $>2.3$ ).

middle frontal gyrus and bilateral putamen and the left caudate nucleus after errors, which again partly overlapped with the FPCN (Fig. 5C and Supplementary Table 4).

Successful response inhibition is associated with similar brain activity regardless of the level of performance monitoring

The neural correlates of successful response inhibition, rather than error processing, were studied by comparing correct stop-trials with go-trials. The high- and low-PM groups both showed increased activity in the posterior cingulate cortex/precuneus compared with control subjects. However, there was no significant difference in activity between patient groups. Comparison of gotrials to rest-trials revealed a predictable pattern of brain activation with bilateral sensory, motor, and superior parietal regions, as well as in the supplementary motor area, occipital regions and the putamen. Task related activation was similar for both patient groups and control subjects, no differences were seen on wholebrain statistical comparison between the groups, suggesting an absence of general, non-specific changes in brain physiology after TBI.

\section{Lesion location and extent does not relate to performance-monitoring deficits}

There was no obvious relationship between the location or extent of focal injuries and performance-monitoring ability. There were few locations where more than two patients in either group had common focal lesions (Fig. 6). Therefore formal statistical analysis of the relationship between lesion location and the likelihood of impaired performance monitoring was not possible. In the highPM group the greatest overlap was $<1 \mathrm{~cm}^{3}\left(964 \mathrm{~mm}^{3}\right)$, centred in the orbitofrontal cortex (peak $-2,-54,-26$ ), where $12.5 \%$ $(5 / 40)$ of the subjects shared a lesion. In the low-PM group the greatest overlap was $\sim 3 \mathrm{~cm}^{3}\left(3005 \mathrm{~mm}^{3}\right)$, centred in the right inferior temporal gyrus (peak $52,-59,-4)$, where $8.7 \%(2 / 23)$ of the subjects shared a lesion. There was no difference in lesion volume between groups (low-PM $12960 \mathrm{~mm}^{3}$ versus high-PM $\left.19634 \mathrm{~mm}^{3} ; P=0.67\right)$. We also used diffusion tensor imaging measures of white matter integrity to investigate whether the fractional anisotropy of tracts connecting key regions of the FPCN and visual network were different between groups (i.e. dorsal anterior cingulate cortex to left and right anterior insulae, and bilateral precuneus to occipital cortex). In both patient groups, all tracts showed significantly greater white matter damage than control subjects. However, no tracts showed significantly different fractional anisotropy values between high- and low-PM groups.

\section{Subanalysis of moderate/severe patients only}

To exclude the possibility that wide variations in injury severity confounded any of the results, we repeated the analyses using only moderate/severe patients. This did not affect the composition of the low-PM group, but resulted in the exclusion of seven highPM patients. The re-analysis did not significantly alter any of the results (Supplementary material).

\section{Discussion}

The neural basis for awareness has been unclear. Impaired awareness is common after $\mathrm{TBI}$, and here we show that low self-awareness is associated with dysfunction within the FPCN. Awareness is likely to be an emergent property of interactions within large-scale distributed brain networks. In keeping with this hypothesis, we found that patients with impaired self-awareness showed reduced functional connectivity from the dorsal anterior cingulate cortex and other frontal regions to the rest of the FPCN. This change was accompanied by an abnormal response to errors within the anterior insulae, which are normally tightly linked to the dorsal anterior cingulate cortex. The results are in keeping with the proposal that interactions between the anterior insulae and dorsal anterior cingulate cortex support the internal monitoring important for self-awareness. 


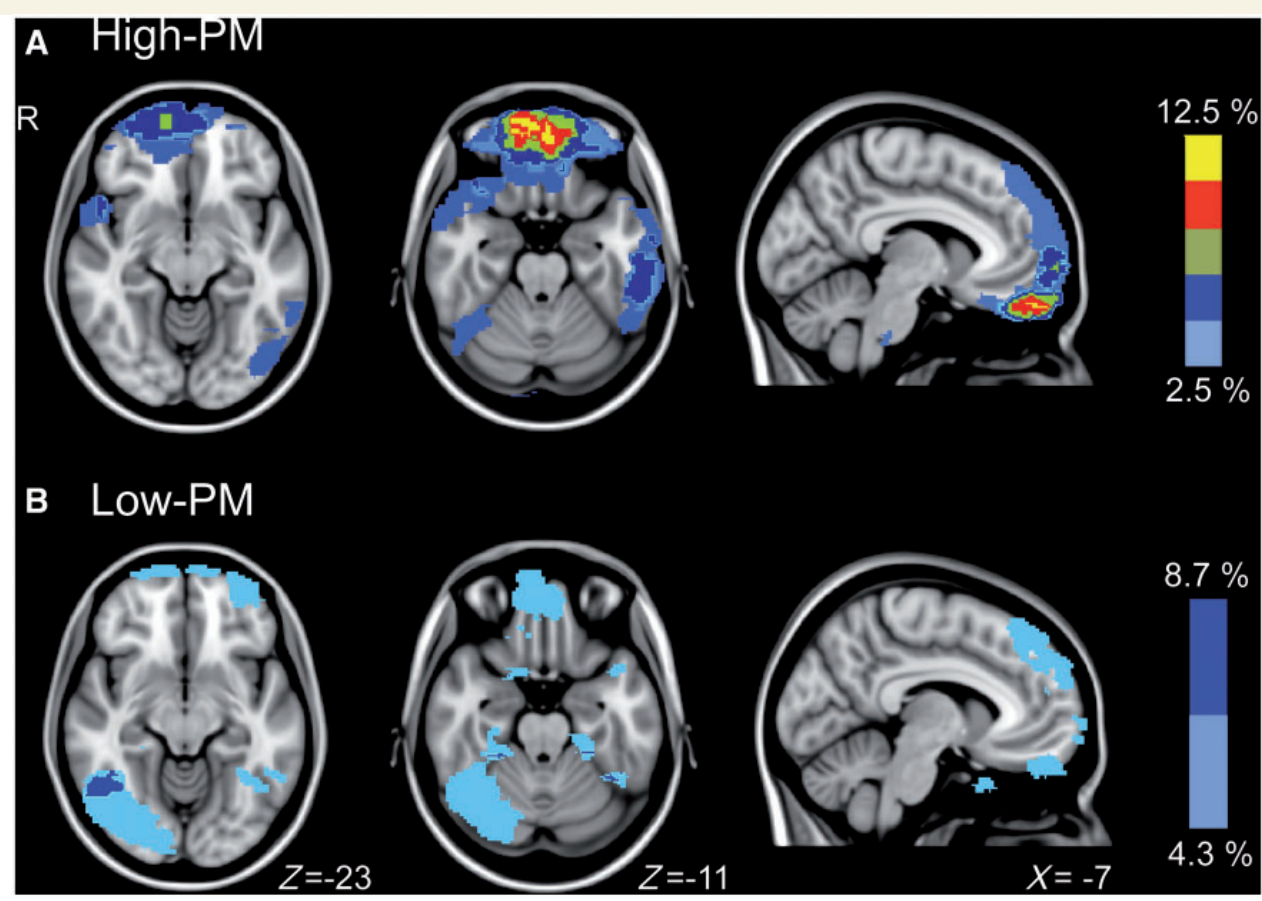

Figure 6 Manually defined lesion overlap maps for high-PM subjects (A), and low-PM subjects (B).

The functional abnormalities we observed within the anterior insulae and dorsal anterior cingulate cortex suggest that self-awareness may be particularly dependent on a sub-network within the FPCN, the salience network. A large body of literature supports an important role for the salience network in performance-monitoring (Oliveira et al., 2007), error detection (Ullsperger et al., 2010; Ham et al., 2012, 2013), and self-reflection (Johnson et al., 2002). Behaviourally salient events usually enter conscious awareness, and it has been proposed that the activity of the anterior insulae and its connections to the dorsal anterior cingulate cortex provide a neural substrate for awareness (Craig, 2009). In this model the dorsal anterior cingulate cortex and insulae interact to continually update ones representation of self. Our results are broadly in keeping with this as we have demonstrated a 'baseline' reduction in functional connectivity within the FPCN of low-PM patients. In particular, the dorsal anterior cingulate cortex is less coupled to other parts of the network including the anterior insulae.

Behaviourally important events normally trigger a tightly coupled response within the salience network, and the right anterior insulae seems to provide the networks driving input (Sridharan et al., 2008; Ham et al., 2013). Actions occur in the context of background brain activity, and these background fluctuations have been shown to influence behavioural responses and associated brain activity (Fox et al., 2006; Sharp et al., 2011), as well as to bias perceptual processing (Boly et al., 2007; Hesselmann et al., 2008). Therefore, it is not unexpected that abnormalities in the 'baseline' functional connectivity of one node of the FPCN might be associated with phasic abnormalities within another part of the network. At first glance an increased phasic activation in response to errors in the impaired patients may seem unexpected. However, evoked activation is often greater in a damaged system. In other contexts increased event-related functional MRI responses have been related to impaired or inefficient neural processing (Baltes, 1993; Cabeza et al., 2002; Ward et al., 2006). Therefore, we speculate that in the low-PM group: (i) there is a degree of sustained functional disconnection within the salience network, measured by the 'resting' functional connectivity; (ii) this results in inefficiency in the interaction between nodes in the network, including the anterior insulae and dorsal anterior cingulate cortex; leading to (iii) abnormally high phasic responses to within the anterior insulae to errors, which we have previously shown to provide the input to the salience network (Ham et al., 2013).

An important caveat in interpreting our event-related analysis of error processing is that it is unclear whether subjects were aware of their errors on the stop-signal task. We used the stop-change task to define patient groups because error correction on this task provides an explicit indication of awareness. However, for methodological reasons we used the stop-signal task, which is a simpler version of the stop-change task, in the neuroimaging part of the study. The stop-signal task does not involve an explicit correction of errors, but has the advantage that it provides a measure of performance monitoring not complicated by the initiation and execution of a second motor response. An indication that low-PM patients were aware of at least a proportion of the errors on the stop-signal task is provided by the post-error slowing data. When errors on the stop-change task were not rapidly corrected there was no post-error slowing, supporting our inference that the subjects were not aware of these errors. In contrast, when stopchange task errors were rapidly corrected, there was abnormally large post-error slowing. This demonstrates that subjects were 
capable of making adjustments to their behaviour, and that subjects were generally engaged by the task. During the stop-signal task post-error slowing was increased overall. This suggests that subjects were aware of at least a proportion of the errors they made. Therefore, it is likely that increased anterior insulae activity in response to errors in the low-PM group is related to inefficient processing of errors that, at least in some cases, reach conscious awareness.

In patients with preserved performance monitoring (high-PM group) we also observed abnormally high activity in the right middle frontal gyrus following errors. This enhanced activation may represent a 'top-down' attentional compensation that the low-PM patients might be unable to engage. In the context of spatial neglect patients, the deficit can be overcome, at least temporarily, by both externally (Robertson et al., 1998) and internally signalled changes in attention (Soto et al., 2009). The increased activity observed in the FPCN may be the neurological correlate of this top-down attentional drive in the high-PM group. In the lowPM group, prolonged reaction times and increased behavioural variability indicate broad attentional problems in addition to impaired performance monitoring. These general problems with engaging or maintaining attention may interact with more specific problems with performance monitoring to produce the behavioural deficits we observed.

We defined our patient groups on their ability to rapidly correct errors. Although this only measures one facet of self-awareness, low-PM patients also showed evidence of more generally impaired awareness. Self-awareness has been quantified in a number of ways (Fleming et al., 1996; O'Keeffe et al., 2007). Questionnaires have been widely used to identify discrepancies between assessments of disability made by the patient and others, providing a 'meta-cognitive' measure of self-awareness (O'Keeffe et al., 2007). Although useful, this type of assessment is limited by its subjective nature and the inconsistency of observer evaluations (Fleming et al., 1996). To control for these problems we used an 'on-line' measure of performance monitoring as the primary objective measure self-awareness (Hart et al., 1998; O'Keeffe et al., 2004). On-line measures of this type have previously been shown to variably relate to other measures of selfawareness such as questionnaire assessments (Hart et al., 1998; O'Keeffe et al., 2004, 2007). In our study, low-PM patients also showed evidence of 'metacognitive' impairments of awareness, which impacted on their behaviour.

Using the Mayo classification of TBI, all patients in the low-PM group had moderate/severe TBI. This classification system incorporates neuroimaging data, and as a result some of the low-PM patients would have been labelled as mild TBI using a more traditional approach to classification. The failure to monitor behaviour efficiently can be a disabling problem that influences many facets of recovery from TBI. For example, patients with impaired performance monitoring can be particularly difficult to rehabilitate (Sherer et al., 2005), as rehabilitation involves learning or re-learning skills, which requires accurate monitoring and appropriate behavioural adaptation in response to errors. In a related way, impairments of self-awareness also contribute to diminished capacity, which is common after TBI (Dreer et al., 2012; Martin et al., 2012). A failure to appreciate ones disability will lead to an impaired ability to make accurate judgements, diminishing patients' capacity (Triebel et al., 2012). Because of its widespread impact, understanding the neural basis for self-awareness impairments are clinically relevant, and may ultimately allow neural measures to complement behavioural assessments in these areas.

The differences between our patient groups were not the result of differences in the amount of structural brain injury. We did not find a relationship between impaired performance monitoring and either patterns of focal brain damage or more diffuse white matter injury. White matter damage was assessed using diffusion tensor imaging, which is a sensitive technique for investigating traumatic axonal injury (Sharp and Ham, 2011). As expected, our patients showed evidence of traumatic axonal injury, but contrary to our predictions this did not explain the performance-monitoring impairment. In particular, there was no relationship between the functional connectivity between FPCN nodes and structural damage to the white matter tracts connecting these nodes. We have previously shown that damage to the tract connecting the right anterior insulae to the dorsal anterior cingulate cortex/ pre-supplementary motor area is associated with attentional impairment (Bonnelle et al., 2012) but in the case of performancemonitoring we observed no significant relationship. This could be because our techniques are not sensitive enough to identify subtle damage to these tracts, or perhaps because impairments of selfawareness emerge in a complex way from the interactions of multiple brain networks and reflect the integrity of a large number of white matter tracts (Thompson and Varela, 2001; Ham and Sharp, 2012). Multivariate approaches to analysing traumatic axonal injury may be well suited to identifying these complex relationships (Hellyer et al., 2012). It is likely to be informative in future work to take a more comprehensive approach to analysing the structure/function relationships underlying impairments of awareness, perhaps starting from a point of equivalent dimensionality between the two modalities.

There are some potential limitations to our study. First, some of our patients with $\mathrm{TBI}$ had focal cortical lesions that might have affected the registration process required to compare across individuals. However, this is unlikely to have produced major errors in our analysis. In general, patients did not have large lesions and the results of registration were checked carefully, resulting in the exclusion of two subjects. In addition, masks of the lesion location were used to improve registration (Brett et al., 2001) and lesion masks were used as a covariate in the functional MRI analyses. A second potential limitation is that we focused the 'resting' state and diffusion tensor imaging analyses on the connections of the FPCN, using the visual network as a control. This choice was motivated by large amounts of literature, suggesting that the dorsal anterior cingulate cortex and anterior insulae are critical for performance monitoring (Carter et al., 1998; Botvinick et al., 2004). In keeping with a key role for these regions in performance monitoring, the event-related analysis of error response, which was not constrained to the FPCN, also showed abnormalities within the anterior insulae. However, future work should investigate in more detail the potential contribution of regions outside the FPCN to impairments of self-awareness.

In summary, we have used detailed behavioural assessment and multi-modal neuroimaging to investigate the neural basis of 
impaired self-awareness after TBI. Functional abnormality with the FPCN is associated with impaired performance-monitoring. This finding is consistent with the principle that awareness is an emergent property of multiple brain regions, and that intact connectivity of the dorsal anterior cingulate cortex and anterior insulae are necessary for rapid error processing.

\section{Funding}

This work was supported by grants from the Medical Research Council, the National Institute of Health Research and the Imperial College Healthcare Charity (to D.J.S).

\section{Supplementary material}

Supplementary material is available at Brain online.

\section{References}

Baltes PB. The aging mind: potential and limits. Gerontologist 1993; 33: 580-94.

Beckmann CF, DeLuca M, Devlin JT, Smith SM. Investigations into resting-state connectivity using independent component analysis. Philos Trans R Soc Lond B Biol Sci 2005; 360: 1001-13.

Bekker EM, Overtoom CC, Kenemans JL, Kooij JJ, De Noord I, Buitelaar JK, et al. Stopping and changing in adults with ADHD. Psychol Med 2005; 35: 807-16.

Boly M, Balteau E, Schnakers C, Degueldre C, Moonen G, Luxen A, et al. Baseline brain activity fluctuations predict somatosensory perception in humans. Proc Natl Acad Sci USA 2007; 104: 12187-92.

Bonnelle $V$, Ham TE, Leech $R$, Kinnunen KM, Mehta MA, Greenwood RJ, et al. Salience network integrity predicts default mode network function after traumatic brain injury. Proc Natl Acad Sci USA 2012; 109: 4690-5.

Bonnelle $V$, Leech $R$, Kinnunen KM, Ham TE, Beckmann CF, De Boissezon $X$, et al. Default mode network connectivity predicts sustained attention deficits after traumatic brain injury. J Neurosci 2011; 31: 13442-51.

Botvinick MM, Cohen JD, Carter CS. Conflict monitoring and anterior cingulate cortex: an update. Trends Cogn Sci 2004; 8: 539-46.

Brett $M$, Leff AP, Rorden C, Ashburner J. Spatial normalization of brain images with focal lesions using cost function masking. Neuroimage 2001; 14: 486-500.

Cabeza R, Anderson ND, Locantore JK, McIntosh AR. Aging gracefully: compensatory brain activity in high-performing older adults. Neuroimage 2002; 17: 1394-402.

Carter CS, Braver TS, Barch DM, Botvinick MM, Noll D, Cohen JD. Anterior cingulate cortex, error detection, and the online monitoring of performance. Science 1998; 280: 747-9.

Corbetta M, Shulman GL. Spatial neglect and attention networks. Annu Rev Neurosci 2011; 34: 569-99.

Craig AD. How do you feel-now? The anterior insula and human awareness. Nat Rev Neurosci 2009; 10: 59-70.

Damoiseaux JS, Rombouts SA, Barkhof F, Scheltens P, Stam CJ, Smith $S M$, et al. Consistent resting-state networks across healthy subjects. Proc Natl Acad Sci USA 2006; 103: 13848-53.

Dehaene S, Posner MI, Tucker DM. Localization of a Neural System for Error-Detection and Compensation. Psychol Sci 1994; 5: 303-5.

Dreer LE, Devivo MJ, Novack TA, Marson DC. Financial capacity following traumatic brain injury: a six-month longitudinal study. Rehabil Psychol 2012; 57: 5-12.
Filippini N, Maclntosh BJ, Hough MG, Goodwin GM, Frisoni GB, Smith $S M$, et al. Distinct patterns of brain activity in young carriers of the APOE-epsilon4 allele. Proc Natl Acad Sci USA 2009; 106: 7209-14.

Fleming JM, Strong J, Ashton R. Self-awareness of deficits in adults with traumatic brain injury: how best to measure? Brain Inj 1996; 10: 1-15.

Fox MD, Snyder AZ, Zacks JM, Raichle ME. Coherent spontaneous activity accounts for trial-to-trial variability in human evoked brain responses. Nat Neurosci 2006; 9: 23-5.

Grace J, Stout JC, Malloy PF. Assessing frontal lobe behavioral syndromes with the frontal lobe personality scale. Assessment 1999; 6: 269-84.

Ham T, Leff A, de Boissezon X, Joffe A, Sharp DJ. Cognitive control and the salience network: an investigation of error processing and effective connectivity. J Neurosci 2013; 33: 7091-8.

Ham TE, de Boissezon X, Leff A, Beckmann C, Hughes E, Kinnunen KM, et al. Distinct frontal networks are involved in adapting to internally and externally signaled errors. Cereb Cortex 2012; 23: 703-13.

Ham TE, Sharp DJ. How can investigation of network function inform rehabilitation after traumatic brain injury? Curr Opin Neurol 2012; 25 662-9.

Hart T, Giovannetti T, Montgomery MW, Schwartz MF. Awareness of errors in naturalistic action after traumatic brain injury. J Head Trauma Rehab 1998; 13: 16-28.

He BJ, Snyder AZ, Vincent JL, Epstein A, Shulman GL, Corbetta $M$. Breakdown of functional connectivity in frontoparietal networks underlies behavioral deficits in spatial neglect. Neuron 2007; 53: 905-18.

Hellyer PJ, Leech R, Ham TE, Bonnelle V, Sharp DJ. Individual prediction of white matter injury following traumatic brain injury. Ann Neurol 2013; 73: 489-9.

Hesselmann G, Kell CA, Eger E, Kleinschmidt A. Spontaneous local variations in ongoing neural activity bias perceptual decisions. Proc Natl Acad Sci USA 2008; 105: 10984-9.

Husain M, Shapiro K, Martin J, Kennard C. Abnormal temporal dynamics of visual attention in spatial neglect patients. Nature 1997; 385 : 154-6.

Jenkinson $M$, Bannister $P$, Brady $M$, Smith $S$. Improved optimization for the robust and accurate linear registration and motion correction of brain images. Neuroimage 2002; 17: 825-41.

Jenkinson $M$, Smith $S$. A global optimisation method for robust affine registration of brain images. Med Image Anal 2001; 5: 143-56.

Johnson SC, Baxter LC, Wilder LS, Pipe JG, Heiserman JE, Prigatano GP. Neural correlates of self-reflection. Brain 2002; 125: 1808-14.

Larson MJ, Kaufman DA, Schmalfuss IM, Perlstein WM. Performance monitoring, error processing, and evaluative control following severe TBI. J Int Neuropsych Soc 2007; 13: 961-71.

Li CS, Huang C, Yan P, Paliwal P, Constable RT, Sinha R. Neural correlates of post-error slowing during a stop signal task: a functional magnetic resonance imaging study. J Cognitive Neurosci 2008; 20: 1021-9.

Logan GD, Cowan WB, Davis KA. On the ability to inhibit simple and choice reaction time responses: a model and a method. J Exp Psychol Human 1984; 10: 276-91.

Malec JF, Brown AW, Leibson CL, Flaada JT, Mandrekar JN, Diehl NN, et al. The mayo classification system for traumatic brain injury severity. J Neurotraum 2007; 24: 1417-24.

Martin RC, Triebel K, Dreer LE, Novack TA, Turner C, Marson DC. Neurocognitive predictors of financial capacity in traumatic brain injury. J Head Trauma Rehab 2012; 27: E81-90.

Mesulam MM. Large-scale neurocognitive networks and distributed processing for attention, language, and memory. Ann Neurol 1990; 28: 597-613.

Modirrousta $M$, Fellows LK. Dorsal medial prefrontal cortex plays a necessary role in rapid error prediction in humans. J Neurosci 2008; 28: 14000-5. 
Oakes TR, Fox AS, Johnstone T, Chung MK, Kalin N, Davidson RJ. Integrating VBM into the General Linear Model with voxelwise anatomical covariates. Neuroimage 2007; 34: 500-8.

O'Keeffe F, Dockree P, Moloney P, Carton S, Robertson IH. Awareness of deficits in traumatic brain injury: a multidimensional approach to assessing metacognitive knowledge and online-awareness. J Int Neuropsych Soc 2007; 13: 38-49.

O'Keeffe FM, Dockree PM, Robertson IH. Poor insight in traumatic brain injury mediated by impaired error processing? Evidence from electrodermal activity. Brain Res Cogn Brain Res 2004; 22: 101-12.

Oliveira FT, McDonald JJ, Goodman D. Performance monitoring in the anterior cingulate is not all error related: expectancy deviation and the representation of action-outcome associations. J Cognitive Neurosci 2007; 19: 1994-2004.

Ornstein TJ, Levin HS, Chen S, Hanten G, Ewing-Cobbs L, Dennis M, et al. Performance monitoring in children following traumatic brain injury. J Child Psychol Psyc 2009; 50: 506-13.

Prigatano GP, Altman IM. Impaired awareness of behavioral limitations after traumatic brain injury. Arch Phys Med Rehabil 1990; 71: 1058-64.

Rabbitt PM. Errors and error correction in choice-response tasks. J Exp Psychol 1966; 71: 264-72.

Rabbitt PM. Three kinds of error-signalling responses in a serial choice task. Q J Exp Psychol 1968; 20: 179-88.

Reid-Arndt SA, Nehl C, Hinkebein J. The Frontal Systems Behaviour Scale (FrSBe) as a predictor of community integration following a traumatic brain injury. Brain Inj 2007; 21: 1361-9.

Ridderinkhof KR, Ullsperger M, Crone EA, Nieuwenhuis S. The role of the medial frontal cortex in cognitive control. Science 2004; 306: 443-7.

Robertson IH, Mattingley JB, Rorden C, Driver J. Phasic alerting of neglect patients overcomes their spatial deficit in visual awareness. Nature 1998; 395: 169-72.

Scheid R, Preul C, Gruber O, Wiggins C, von Cramon DY. Diffuse axonal injury associated with chronic traumatic brain injury: evidence from T2* -weighted gradient-echo imaging at $3 \mathrm{~T}$. Am J Neuroradiol 2003; 24: 1049-56.

Schmitz TW, Rowley HA, Kawahara TN, Johnson SC. Neural correlates of self-evaluative accuracy after traumatic brain injury. Neuropsychologia 2006; 44: 762-73.

Seeley WW, Menon V, Schatzberg AF, Keller J, Glover GH, Kenna H, et al. Dissociable intrinsic connectivity networks for salience processing and executive control. J Neurosci 2007; 27: 2349-56.

Sharp DJ, Beckmann CF, Greenwood R, Kinnunen KM, Bonnelle V, De Boissezon $X$, et al. Default mode network functional and structural connectivity after traumatic brain injury. Brain 2011; 134: 2233-47.

Sharp DJ, Bonnelle V, De Boissezon X, Beckmann CF, James SG, Patel MC, et al. Distinct frontal systems for response inhibition, attentional capture, and error processing. Proc Natl Acad Sci USA 2010; 107: 6106-11.

Sharp DJ, Ham TE. Investigating white matter injury after mild traumatic brain injury. Curr Opin Neurol 2011; 24: 558-63.

Sharp DJ, Scott SK, Mehta MA, Wise RJ. The neural correlates of declining performance with age: evidence for age-related changes in cognitive control. Cereb Cortex 2006; 16: 1739-49.

Sherer M, Hart T, Nick TG, Whyte J, Thompson RN, Yablon SA. Early impaired self-awareness after traumatic brain injury. Arch Phys Med Rehabil 2003; 84: 168-76.
Sherer M, Hart T, Whyte J, Nick TG, Yablon SA. Neuroanatomic basis of impaired self-awareness after traumatic brain injury: findings from early computed tomography. J Head Trauma Rehabil 2005; 20: 287-300.

Smith SM, Fox PT, Miller KL, Glahn DC, Fox PM, Mackay CE, et al. Correspondence of the brain's functional architecture during activation and rest. Proc Natl Acad Sci USA 2009; 106: 13040-5.

Smith SM, Jenkinson $M$, Johansen-Berg $H$, Rueckert $D$, Nichols TE, Mackay $C E$, et al. Tract-based spatial statistics: voxelwise analysis of multi-subject diffusion data. Neuroimage 2006; 31: 1487-505.

Smith SM, Jenkinson M, Woolrich MW, Beckmann CF, Behrens TE, Johansen-Berg $\mathrm{H}$, et al. Advances in functional and structural $M R$ image analysis and implementation as FSL. Neuroimage 2004; 23 (Suppl 1): S208-19.

Soto D, Funes MJ, Guzman-Garcia A, Warbrick T, Rotshtein P, Humphreys GW. Pleasant music overcomes the loss of awareness in patients with visual neglect. Proc Natl Acad Sci USA 2009; 106: 6011-6.

Spreng RN. The fallacy of a 'task-negative' network. Front Psychol 2012; 3: 145.

Spreng RN, Stevens WD, Chamberlain JP, Gilmore AW, Schacter DL. Default network activity, coupled with the frontoparietal control network, supports goal-directed cognition. Neuroimage 2010; 53: 303-17.

Squarcina L, Bertoldo A, Ham TE, Heckemann R, Sharp DJ. A robust method for investigating thalamic white matter tracts after traumatic brain injury. Neuroimage 2012; 63: 779-88.

Sridharan D, Levitin DJ, Menon V. A critical role for the right frontoinsular cortex in switching between central-executive and defaultmode networks. Proc Natl Acad Sci USA 2008; 105: 12569-74.

Taylor JG. Neural networks for consciousness. Neural Netw 1997; 10 1207-25.

Thompson E, Varela FJ. Radical embodiment: neural dynamics and consciousness. Trends Cogn Sci 2001; 5: 418-25.

Triebel KL, Martin RC, Novack TA, Dreer L, Turner C, Pritchard PR, et al. Treatment consent capacity in patients with traumatic brain injury across a range of injury severity. Neurology 2012; 78: 1472-8.

Ullsperger M, Harsay HA, Wessel JR, Ridderinkhof KR. Conscious perception of errors and its relation to the anterior insula. Brain Struct Funct 2010; 214: 629-43.

Ullsperger $M$, von Cramon DY. Neuroimaging of performance monitoring: error detection and beyond. Cortex 2004; 40: 593-604.

Vanderploeg RD, Belanger HG, Duchnick JD, Curtiss G. Awareness problems following moderate to severe traumatic brain injury: prevalence, assessment methods, and injury correlates. J Rehabil Res Dev 2007; 44: 937-50.

Verbruggen F, Logan GD. After-effects of goal shifting and response inhibition: a comparison of the stop-change and dual-task paradigms. Q J Exp Psychol 2008; 61: 1151-9.

Vincent JL, Kahn I, Snyder AZ, Raichle ME, Buckner RL. Evidence for a frontoparietal control system revealed by intrinsic functional connectivity. J Neurophysiol 2008; 100: 3328-42.

Ward NS, Newton JM, Swayne OB, Lee L, Thompson AJ, Greenwood RJ, et al. Motor system activation after subcortical stroke depends on corticospinal system integrity. Brain 2006; 129: 809-19.

Zuo XN, Kelly C, Adelstein JS, Klein DF, Castellanos FX, Milham MP. Reliable intrinsic connectivity networks: test-retest evaluation using ICA and dual regression approach. Neuroimage 2010; 49: 2163-77. 